\title{
Preface
}

\section{The Second Symposium on Innovative Polymers for Controlled Delivery, September 11-14, 2012, Suzhou, China}

We are witnessing a rapid development of innovative polymer-based biomaterials for controlled delivery of therapeutics and biotherapeutics (e.g., drugs, proteins, siRNA and DNA), targeted delivery of diagnostic agents (e.g., quantum dots and iron oxides), as well as functional delivery of cells and/or bioactives (e.g., stem cells and growth factors) in tissue engineering and regenerative medicine. It is interesting to note that innovation in delivery systems has already made significant technological impacts on medical research and practice. The Second Symposium on Innovative Polymers for Controlled Delivery (SIPCD 2012) was held in September 11-14, 2012 in Suzhou, China, to provide a high-level platform for worldwide top scientists, entrepreneurs, prominent young researchers and graduate students to meet and discuss state-of-the-art progress, challenging issues and future perspectives of controlled release systems. To our great pleasure, SIPCD 2012 has received overwhelming attention from different disciplines including polymers, biomedical engineering, pharmacy, nanotechnology, and regenerative medicine. The following block gives an overview of the highlights of the SIPCD 2012 symposium.

Highlights of SIPCD 2012 Symposium

30 invited lectures by leading researchers including editors and associate editors of over 10 prestigious journals

- 252 poster presentations selected out of over 300 submissions

418 delegates from 138 institutions out of 18 countries

intriguing panel discussion on "What is The Future of Nanomedicine?"

an Author Workshop on "The Art of Writing Great Scientific Papers"

Eublication of a special issue of the Journal of Controlled Release (Elsevier)

on line publication of poster abstracts in the Journal of Controlled Release

Biomacromolecules Best Poster Awards (ACS)

I. conference report in Nanomedicine (Future Science Group)

As the First Symposium on Innovative Polymers for Controlled Delivery (SIPCD 2010) [1], SIPCD 2012 presented a high quality scientific program with 30 invited lectures given by selected worldwide leading scientists including editors and associate editors of over 10 prestigious journals. A flavor of the SIPCD 2012 symposium can be perceived from our conference report published in Nanomedicine [2] as well as the twenty-two peer-reviewed articles contributed by our invited speakers in this special issue of the Journal of Controlled Release. We appreciate the kind collaboration of Wim E. Hennink and Kinam Park in making such a quality special issue that will certainly attract broad interest from readers. In SIPCD 2012, we also hosted an intriguing panel discussion on "What is The Future of Nanomedicine?" led by David W. Grainger (Chair), Frank Caruso, Si-Shen Feng, and Wim E. Hennink as well as an interesting Author Workshop on "The Art of Writing Great Scientific Papers" by Jaap van Harten (Executive Publisher, Elsevier). Moreover, 252 poster presentations were accepted from more than 300 submissions following a strict peer-review process. Selected by the Best Poster Awards Committee constituted with Harm-Anton Klok (Chair), Jiandong Ding, Johan F. J. Engbersen, Phillip B. Messersmith, Stefaan de Smedt and Hsing-Wen Sung, six poster presenters have received prestigious Biomacromolecules Poster Awards (ACS). The abstracts of all posters presented at SIPCD 2012 symposium will be made available online in this special issue of the Journal of Controlled Release.

The symposium was co-organized by Soochow University, Changchun Institute of Applied Chemistry of the Chinese Academy of Sciences, Suzhou Industrial Park and Suzhou BioBay. We highly appreciate our organizing committee members in particular Xuesi Chen, Yuwen Liu, Cheolho Kwak, Harm-Anton Klok, Jaap van Harten, Kazunori Kataoka, Kinam Park, Wim E. Hennink, Jin Ma, Xiulin Zhu, and Hong Chen for their valuable contributions. We would like to extend our appreciation to the enthusiastic cooperation and support of our sponsors including the National Natural Science Foundation of China (NSFC), Elsevier, Ssens B.V., NOF Corporation, American Chemical Society (ACS), Samyang Corporation, Suzhou Industrial Park, Priority Academic Program Development of Jiangsu Higher Education Institutions, Changchun Institute of Applied Chemistry of the Chinese Academy of Sciences, Jiangsu Society of Chemistry and Chemical Engineering, Advanced Academic Exchange Activities of Suzhou, Malvern Instruments, Jiangsu Key Laboratory of Advanced Functional Polymer Design and Application (Soochow University), Suzhou BioBay, as well as two media partners (biomat.net and polymer.cn) that made this unique symposium possible. We are thankful to our colleagues (in particular Ru Cheng, Chao Deng, and Fenghua Meng) and students from Soochow University Biomedical Polymers Laboratory for their devoted volunteer work. Together we succeeded in making this SIPCD 2012 a scientifically rewarding and socially enjoyable event.

Here, we are pleased to announce that The Third Symposium on Innovative Polymers for Controlled Delivery (SIPCD 2014) will be held in September 2014 in Suzhou, China. SIPCD 2014 will be a most special event in that it is dedicated to Prof. Jan Feijen for his 
70th birthday. For further information, please contact the SIPCD secretariat (E-mail: sipcd@suda.edu.cn) or check out the symposium homepage (http://www.sipcd.cn). We look forward to welcoming you to SIPCD 2014.

\section{References}

[1] Z. Zhong, J. Feijen, The first symposium on innovative polymers for controlled delivery, September 14-17, 2010, Suzhou, China, J. Control. Release 152 (2011) 1-1.

[2] J. Feijen, W.E. Hennink, Z. Zhong, Conference scene: from innovative polymers to advanced nanomedicine: key challenges, recent progress and future perspectives, Nanomedicine 8 (2013) 177-180.
Zhiyuan Zhong* Jan Feijen

Biomedical Polymers Laboratory, Jiangsu Key Laboratory of Advanced Functional Polymer Design and Application, College of Chemistry, Chemical Engineering and Materials Science, Soochow University, Suzhou 215123, PR China

*Corresponding author. Tel./fax: + 8651265880098

E-mail addresses: zyzhong@suda.edu.cn (Z. Zhong) j.feijen@utwente.nl (J. Feijen) 